\title{
Mean platelet volume in patients with periodontal disease with or without acute vascular events Enperiva II
}

\begin{abstract}
During the last 15 years, periodontal disease has started to be regarded as a predictor of atheromatous disease. The hypothesis of chronic inflammation stimulating mediators which accelerate endothelial dysfunction may explain the association between these two pathologies. Both of them could also increase platelet response and adhesion, which can be easily accessed by determining mean platelet volume, an indicator of higher platelet reactivity. Considering the facts mentioned above, we propose a case-control study to establish the relation among subjects with periodontal disease suffering major vascular events and to relate these conditions with mean platelet volume. A case-control study was conducted. A total number of 30 cases and 32 controls were included consecutively. The presence of periodontal disease was higher in the case group than in the control group $(70 \%$ vs. $40 \% ; \mathrm{p}=0.02)$. When cases with periodontal disease and controls with periodontal disease were compared, we found that mean platelet volume (MPV) was significantly higher in the latter $(\mathrm{p}=0.01)$
\end{abstract}

Keywords: periodontal disease, periodontitis, vascular events, acute coronary syndrome, mean platelet volume
Volume 9 Issue I - 2018

\author{
Pablo A Olavegogeascoechea, ${ }^{1,2}$ Alberto \\ Grandinetti, ${ }^{2}$ Fernando Lipovestky, \\ Alejandro Schroeder, ${ }^{3}$ Sergio Perez, ${ }^{3}$ Nicol \\ s Perez, ${ }^{3}$ Pablo Contreras, ${ }^{3}$ Maria I Brusca ${ }^{2}$ \\ 'Department of Collective Health, National University of \\ Comahue, Argentina \\ ${ }^{2} S c h o o l$ of Dentistry, Interamerican Open University, Argentina \\ ${ }^{3}$ Department of Cardiology, Medical Foundation of Río Negro \\ and Neuquén, Argentina
}

\begin{abstract}
Correspondence: María I Brusca, School of Dentistry, Interamerican Open University, Buenos Aires, Av. San Juan 95I, CABA,Argentina, Email mariaisabelbrusca@gmail.com
\end{abstract}

Received: January 25, 2017 | Published: February 08, 2018

\section{Introduction}

Vascular disease and the events associated with it are one of the leading causes of morbidity and mortality in adults. ${ }^{1,2}$ Among vascular events, acute coronary syndrome (ACS), represented by a variety of clinical manifestations such as unstable angina and acute myocardial infarction with or without ST-segment elevation, accounts for most events following atheromatous disease complications, i.e. plaque fissuring and rupture followed by intraluminal platelet aggregation., Besides the vulnerability of the atheromatous plaque, these patients show an increased inflammatory state. ${ }^{5,6}$ Traditional risk factors, like hypertension, dyslipidemia, smoking, sedentary lifestyle, obesity and diabetes, have been described since the sixties $;{ }^{7}$ however, new clinical risk factors have been identified since then, e.g. periodontal disease, which has started to be considered as a predictor of atheromatous disease during the last 15 years. ${ }^{8}$ The hypothesis that chronic inflammation stimulates mediators which accelerate endothelial dysfunction ${ }^{9,10}$ and/or mechanisms of cross-reacting antibodies against the periodontal flora and endothelial cell proteins ${ }^{11}$ may explain the connection between these two conditions. Both of them could also increase platelet response and adhesion, which can be easily accessed by determining mean platelet volume (MPV), an indicator of higher platelet reactivity. ${ }^{12} \mathrm{MPV}$ was higher in patients with risk factors and vascular events, ${ }^{13-16}$ as a marker of an inflammatory state. However, MPV was low in patients with periodontitis and it improved after treatment. ${ }^{17}$ This difference could be explained by the fact that the systemic inflammatory state in active periodontal disease is not severe enough to modify MPV. Considering what we have stated so far and the existing disagreement, ${ }^{18}$ the aim of the study is to know the relationship between periodontal disease (periodontitis) and MPV in subjects with acute vascular events such as acute coronary syndrome (ACS), peripheral artery disease (PAD) and Ischemic Stroke (IschS).

\section{Materials and methods}

The design was a case-control study that it was conducted from March to August 2016. The subjects who participated were enrolled in only one Institution. The selection of cases was non-randomized; the participants were patients with acute vascular event, such as acute coronary syndrome (ACS), ischemic stroke (IschS) or acute peripheral artery disease/acute limb ischemia (PAD), and all admitted to the cardiac intensive care unit. Dental evaluation was carried out within $72 \pm 24.8$ hours after admission. Controls were included based on a 1:1 case-control ratio, except for the two last cases, for which a $1: 2$ ratio was used; they were paired according to age ( \pm 5 years), gender and typical risk factors, i.e. hypertension, diabetes, dyslipidemia, family history of coronary artery disease, sedentary lifestyle, current or former smoking habit and BMI $\left( \pm 3 \mathrm{~kg} / \mathrm{m}^{3}\right)$. Given that most patients were exposed to more than one risk factor for vascular disease, the matching was based on those risk factors which have a greater probability of precipitating vascular events according to the Framingham risk score (high blood pressure, diabetes, smoking). ${ }^{19} \mathrm{~A}$ total number of 36 potential participants were recruited; 2 of them refused to participate, 3 were discharged from hospital before dental evaluation and 1 before blood sampling; 32 controls were included. The screening of controls and cases was done contemporaneously; the average interval between the enrollment of a case and the enrollment of its corresponding control was $2.3 \pm 0.7$ weeks. The study was approved by the Research Department and the Ethics Committee at Universidad Abierta Interamericana (Interamerican Open University) 
(PS2/2014) and conducted in accordance with the 1975 Declaration of Helsinki, revised in 1983 and 2013. Before enrolling, participants had to sign an informed consent.

\section{Definitions}

We define ACS (unstable angina, non-ST-segment elevation $\mathrm{MI}^{20}$ or ST-segment elevation MI) as:

1. Unstable angina (UA) is defined as ischemic chest discomfort (or equivalent) that occurs at rest with at least 1 episode lasting $\geq 10$ minutes and is accompanied by new or presumably new STsegment deviation (transient elevation $\geq 0.1 \mathrm{mV}[<20$ minutes $]$ or dynamic horizontal/down-sloping depression $\geq 0.05 \mathrm{mV}$ ) in at least 2 contiguous leads without diagnostic biochemical changes in cardiac enzymes (serum troponin I or T, or creatine kinase-MB).

2. Non-ST-segment elevation MI (NSTEMI) is defined as ischemic chest discomfort (or equivalent) that occurs at rest with at least 1 episode lasting $\geq 10$ minutes and is accompanied by a diagnostic elevation in cardiac biomarkers of myocardial injury (serum troponin I or T, or creatine kinase-MB) above the upper limit of normal without persistent ST-segment elevation.

3. ST-segment elevation MI (STEMI) is defined as prolonged symptoms of ischemic chest discomfort (or equivalent) at rest with at least 1 episode lasting $>20$ minutes and new or presumably new electrocardiographic changes (persistent STsegment elevation $\geq 0.1 \mathrm{mV}$ in $\geq 2$ contiguous precordial leads or $\geq 2$ adjacent limb leads or new LBBB) that are accompanied by a diagnostic elevation in cardiac biomarkers (serum troponin I or $\mathrm{T}$, creatine kinase or creatine kinase-MB) above the upper limit of normal.

a. We define Ischemic Stroke as documented focal neurologic deficit thought to be of vascular origin, with signs or symptoms lasting $>24$ hours. Diagnosis was confirmed by neuroimaging, such as computed tomography (CT) scan or magnetic resonance imaging (MRI). The embolic origin was discarded as the cause of ischemia.

b. Acute peripheral artery disease (APAD) was documented by one of the following: current intermittent claudication with objective evidence of vascular origin, history of peripheral arterial stenting or surgery (including amputation due to vascular causes), or ankle-brachial index $<0.9$ in at least one ankle and the onset of acute limb pain followed by coldness and numbness of the right foot confirmed by angiography. The embolic origin was discarded as the cause of ischemia.

c. Risk factors for vascular disease: family history (FH) of acute ischemic heart disease (any first degree relative [parents, siblings, children] who have had angina, myocardial infarction or sudden death with no apparent cause before the age of 55), diabetes mellitus (history of diabetes, need for oral antidiabetic drugs or insulin use, or fasting blood glucose $>110 \mathrm{mg} / \mathrm{dL}$ ), high blood pressure (HBP) (history of high blood pressure diagnosed and treated with medication, exercise and/or diet; systolic blood pressure $>140 \mathrm{mmHg}$ or diastolic blood pressure $>90 \mathrm{mmHg}$ at least twice; current use of antihypertensive medication), being a smoker (having smoked in the month before admission or having stopped smoking between 1 month and 1 year before inclusion) or a former smoker (having stopped $>1$ year before inclusion), overweight/obesity (BMI $>25 \mathrm{~kg} / \mathrm{m}^{3}$ ), dyslipidemia (history of dyslipidemia diagnosed and/or being treated with hypolipemic agents), sedentary lifestyle (individuals who engage in low-intensity physical activity). ${ }^{21}$

\section{Inclusion criteria}

i. Signature on the informed consent for both groups.

ii. Cases: patients admitted to the intensive care unit with ACS, APAD and/or ischemic stroke aged $>21$; patients who have not taken vitamin or mineral supplements in the last 3 months; presence of at least 7 teeth. Controls: patients admitted to the intensive care unit or in the general ward without acute vascular events, aged $>21$; patients who have not taken vitamin or mineral supplements in the last 3 months; presence of at least 7 teeth.

\section{Exclusion criteria}

patients with cancer of any origin previously diagnosed; patients with cirrhosis, known biliary abnormalities (with the exception of Gilbert's syndrome or asymptomatic gallstones), unstable liver disease or evidence of abnormal liver function tests (total bilirubin or alkaline phosphatase $>1.5$ per upper limit of normal [ULN]; or ALT $>2.5$ per ULN), or other hepatic abnormalities that, in the opinion of the investigator, would preclude the subject from participation in the study; patients with any chronic inflammatory autoimmune diseases; patients undergoing periodontal treatment in the last 3 months; women with positive pregnancy test or known to be pregnant or lactating; patients who have taken antibiotics in the last 3 months.

\section{Dental procedures}

At the beginning of the study, a medical record of each patient was entered. Patients were examined using sterile equipment such as a mouth mirror, an explorer $\mathrm{n}^{\circ} 23$ and a WHO probe. There was no prior antibiotic prophylaxis. Patients were asked not to brush their teeth on the dental evaluation day, but they could use mouthwashes. Oral hygiene and periodontal status were assessed in every tooth, except for third molars, using Silness and Löe plaque index (PI) and gingival index (GI) (1964), ${ }^{22}$ measuring probing depth (PD) and clinical attachment loss (CAL). Four smooth surfaces (vestibular, lingual/palatal, distal and mesial) of 6 representative teeth $(1.6 ; 2.1 ; 2.4 ; 3.6 ; 4.1 ; 4.4)$ were examined. In the case of missing dental pieces, their adjacent tooth, which had the same features, was evaluated. The PI was classified as follows: $0=$ no biofilm; $1=$ no biofilm observed at first sight, but found using a probe on gingival margin; 2=biofilm observed at first sight; $3=$ abundant biofilm. The highest values measured were considered. The GI was classified as follows: $0=$ total absence of signs of inflammation; $1=$ a slight change in color and texture; $2=$ visible signs of inflammation and gingival margin bleeding tendency in contact with the probe, and 3=apparent inflammation with spontaneous bleeding or ulcerations. Periodontal disease spread (PD and CAL) was defined according to the percentage of deep pockets. The mean highest values of PD and CAL for each patient were determined through the division of the deepest pockets values by the total number of deep pockets. Periodontal disease (POD) was defined as the presence of four or more teeth with the highest value of PPD $\geq 3 \mathrm{~mm}$ and $\mathrm{CAL} \geq 3 \mathrm{~mm} .{ }^{22}$ Blood count: automated biochemical analysis systems (Wiener Lab CB 350i and Wiener Lab CM250, Wiener Lab SAIC, Riobamba 2944, Rosario, Argentina) and hematology analysis systems were used. Not all the patients were in a 
fasted state before blood sampling. Samples for measurement of mean platelet volume (MPV) were taken in EDTA tubes and analyzed with an automatic hematology analyzer system (Cell-Dyn Ruby analyzer [Abbott Diagnostics], Abbott Park, IL, USA). Reference values: CKMB: $<25 \mathrm{IU} / \mathrm{L}$; hemoglobin: men $12.5-17 \mathrm{~g} \%$-women $11.5-15 \mathrm{~g} \%$; platelet count: $150000-400000 / \mathrm{mm}^{3}$; plasma glucose: $70-100 \mathrm{mg} / \mathrm{dL}$;

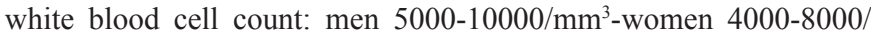
$\mathrm{mm}^{3}$; total cholesterol<200 mg/dL, MPV:6.9-10.6 fl.

\section{Statistical analysis}

In order to determine the sample size, a difference between means of $1 \mathrm{fl}$ and standard deviation of 1.2 was considered, with an alpha error below 0.05 and a power of $80 \%$. Thus, the minimum number of subjects was 30 in each group (assuming at least a 1:1 case-control ratio). The mean values were calculated through a cumulative sum, and to compare those, nonparametric statistics was used: Mann-Whitney $\mathrm{U}$ test, given the number of patients per group. The categorical or dichotomous variables are presented as proportions and compared through $\mathrm{X}^{2}$ test or the Mantel-Haenszel test when necessary. For the univariate analysis of risk factors between groups, unadjusted odds ratio (OR) and $95 \% \mathrm{CI}$ were used. If significant differences were found in the univariate analysis, the multinomial logistic regression would be applied. A significance level of $\mathrm{p}<0.05$ for a two-sided test was assumed. IBM SPSS statistics 22 was used for the statistical analysis.

\section{Results}

Table 1 shows the basal characteristics of both groups. A total number of 62 subjects were included: 30 cases and 32 controls. There were 28 men (93\%) in the case group and $30(93.75 \%)$ in the control group. In the case group, acute vascular events followed acute coronary syndrome (28 subjects) and acute peripheral artery disease (1 subject); 1 patient had posterior circulation ischemic stroke. The average ages were $60.10 \pm 8.93$ for cases and $60.53 \pm 8.97$ for controls $(\mathrm{p}=0.85)$. Smoking habits were registered in $40 \%$ of cases $(n=12)$ and $19 \%$ of controls $(n=6)$, this difference not being significant $(\mathrm{p}=0.15)$. In both groups, the prevalence of the other risk factors was similar: hypertension $(\mathrm{p}=0.77)$, dyslipidemia $(\mathrm{p}=0.45)$, diabetes $(\mathrm{p}=0.59)$, sedentary lifestyle $(\mathrm{p}=0.99)$, former smoking habit $(\mathrm{p}=0.99)$, BMI $(\mathrm{p}=0.76)$, number of teeth $(\mathrm{p}=0.54)$, physical activity $(\mathrm{p}=0.54)$. The combination of risk factors in both groups was highly frequent with no significant difference; 26 cases $(86 \%)$ and 28 controls $(78 \%)$ were exposed to at least 2 risk factors (OR: 0.92; 95 \% CI: 0.21-4.10; $\mathrm{p}=1)$. The most common risk factor association in both groups was between hypertension and overweight/obesity. The proportion of patients with POD was considerably higher in the case group than in the control one $(\mathrm{p}=0.02)$. For this association, the phi coefficient was +0.29 . The most marked difference was observed in patients with moderate POD $(\mathrm{p}=0.05)$; regarding severe periodontitis, cases showed a slightly higher tendency than controls $(\mathrm{p}=0.07)$, and no notable differences were perceived in patients with mild POD $(\mathrm{p}=0.31)$. Presence of gingivitis was equivalent in both groups $(\mathrm{p}=0.99)$. However, the plaque and gingival indexes were significantly higher in cases (PI: $1.74 \pm 0.64$ vs. $0.99 \pm 0.69$, respectively; $\mathrm{p}<0.001$; GI: $1.61 \pm 0.66$ vs. $0.98 \pm 0.55$, respectively; $\mathrm{p}=0.001$ ) (Table 2). Among the cases, the number of affected blood vessels was: 1 vessel $=36.7 \%$; 2 vessels $=20 \%$, and 3 vessels $=33.3$ $\%$. When biochemical and hematological variables were analyzed, only one significant and expected difference was found between the two groups: $\mathrm{CK}-\mathrm{MB}$ plasma levels $(\mathrm{p}=0.007)$. A tendency to higher plasma glucose levels $(\mathrm{mg} / \mathrm{dL})$ was observed in cases $(\mathrm{p}=0.053)$, whereas no important differences were found in total cholesterol, white blood cell count, platelet count, MPV and hemoglobin level, as shown in Table 3. The mean platelet volume in cases compared to controls (whether or not they had POD) showed no significant differences $(8,29 \pm 1,66 \mathrm{fl}$ vs. $8 \pm 1,49 \mathrm{fl}$, respectively; $\mathrm{p}=0,47)$. On the other hand, between patients with acute vascular events (cases) with $(\mathrm{n}=21)$ and without $(\mathrm{n}=9)$ periodontitis, there was not found difference in $\operatorname{MPV}(7,81 \pm 1 \mathrm{fl}$ vs $9,41 \pm 2,34$, respectively; $p=0,16)$. When patients without acute vascular events (controls) were analyzed, an important difference was found between those with periodontal disease and those without $(8.63 \pm 1.90 \mathrm{fl}$ vs. $7.48 \pm 0.91 \mathrm{fl}$, respectively; $\mathrm{p}=0.03)$. Finally, when patients with acute vascular events (cases) with POD and those without acute vascular events (controls) with also POD were compared in relation to MPV, a significant difference was found ( $7.81 \pm 1 \mathrm{fl}$ vs. $9.41 \pm 2.34 \mathrm{fl}$, respectively; $\mathrm{p}=0.01$ ), showing that MPV was higher in patients without acute vascular events who also had concomitant periodontitis.

Table I Baselines at both groups

\begin{tabular}{lllll}
\hline Variables & Cases n (\%) & Controls n (\%) & OR $(95 \%$ Cl) & p $^{*}$ \\
\hline Age (years) & $60.10 \pm 8.93$ & $60.53 \pm 8.97$ & & 0.87 \\
N of T & $18.13 \pm 6.86$ & $22.38 \pm 6.27$ & & 0.54 \\
HT & $17(56 \%)$ & $17(53 \%)$ & $1.15(0.42-3.14)$ & 0.77 \\
DLP & $12(40 \%)$ & $16(50 \%)$ & $0.66(0.24-1.82)$ & 0.45 \\
Dbt & $12(40 \%)$ & $10(31 \%)$ & $1.46(0.51-4.17)$ & 0.59 \\
Sed & $15(50 \%)$ & $16(50 \%)$ & $I(0.36-2.70)$ & 0.99 \\
CSH & $12(40 \%)$ & $6(19 \%)$ & $2.66(0.84-8.46)$ & 0.15 \\
RSH & $12(40 \%)$ & $12(37 \%)$ & $1.11(0.39-3.08)$ & 0.99 \\
FH & $8(26 \%)$ & $6(19 \%)$ & $1.57(0.47-5.23)$ & 0.54 \\
BMI kg $/ \mathrm{m}^{3}$ & $27.31 \pm 4.99$ & $27.79 \pm 6.23$ & & 0.14 \\
\hline
\end{tabular}

*Mann-Whitney $\mathrm{U}$ test was used for independent samples of continuous variables and $\mathrm{x}^{2}$ test for dichotomous variables $\mathrm{N}$ of T, number of teeth; $\mathrm{HT}$, hypertension; DLP, dyslipidemia; Dbt, diabetes mellitus; Sed, sedentary lifestyle; $\mathrm{CSH}$, current smoking habit; RSH, remote smoking habit; BMI, body mass index; FH, family history of acute ischemic heart disease 
Table 2 Periodontitis between groups

\begin{tabular}{lllll}
\hline Variables & Cases n (\%) & Controls n (\%) & OR $(95 \%$ Cl) & $\mathbf{p}^{*}$ \\
\hline POD & $21(70 \%)$ & $13(40 \%)$ & $3.41(1.19-9.76)$ & 0.02 \\
Mild POD & $5(16 \%)$ & $8(25 \%)$ & $0.60(0.17-2.09)$ & 0.53 \\
Moderate POD & $9(30 \%)$ & $3(9 \%)$ & $4.14(0.99-17.17)$ & 0.05 \\
Severe POD & $7(23 \%)$ & $2(6 \%)$ & $4.56(0.86-24.07)$ & 0.07 \\
Gingivitis & $26(86 \%)$ & $27(84 \%)$ & $1.20(0.29-4.98)$ & 0.99 \\
GI & $1.61 \pm 0.66$ & $0.98 \pm 0.55$ & & 0.001 \\
PI & $1.74 \pm 0.64$ & $0.99 \pm 0.69$ & & $<0.001$ \\
\hline
\end{tabular}

*Mann-Whitney $U$ test was used for independent samples of continuous variables and $x^{2}$ test for dichotomous variables

POD, periodontal disease; GI, gingival index; PI, plaque index

Table 3 Biochemical and hematological variables between groups

\begin{tabular}{|c|c|c|c|}
\hline Variables & Cases Media(SD) & Controls Media(SD) & $\mathbf{p}^{*}$ \\
\hline Cholesterol (mg/dl) & $|6| .37 \pm 4 \mid .15$ & $188.97 \pm 47.29$ & 0.22 \\
\hline Glucose (mg/dl) & $\mid 40.80 \pm 57.263$ & $116.03 \pm 35.713$ & 0.053 \\
\hline Leucocytes count (mm3) & $9381.03 \pm 3|7| .80$ & $8573.40 \pm 3040.49$ & 0.31 \\
\hline Hemoglobin (g\%) & $13.64 \pm 2.08$ & $13.58 \pm 1.74$ & 0.90 \\
\hline CK MB (UI/I) & $29.87 \pm 35.86$ & $\mid \mathrm{I} .8 \mathrm{II} \pm 3.48$ & 0.001 \\
\hline Uric Acid (mg/dl) & $4.60 \pm 1.479$ & $5.65 \pm 1.748$ & 0.15 \\
\hline Count Plaques $\left(\mathrm{mm}^{3}\right)$ & $234833.33 \pm 126169.859$ & $213225.81 \pm 67325.928$ & 0.40 \\
\hline MPV (fl) & $8.29 \pm 1.66$ & $8 \pm 1.49$ & 0.47 \\
\hline
\end{tabular}

*Mann-Whitney U-test was used for independent samples

CK MB, creatine phosphokinase MB; MPV, mean platelet volume

\section{Discussion}

In this study, the baseline variables comparation between groups showed no significant differences between both groups, which make the subsequent comparisons relevant. Periodontal disease was found to be more prevalent in subjects with acute vascular events than in those without. This result agrees with previous publications. ${ }^{23-25}$ Prevalence was stronger for moderate periodontitis. The prevalence of POD showed no difference according to the severity of the coronary disease, estimated to have between 1 and 3 blood vessels with occlusions $>70 \%$. On the other hand, plaque and gingival indexes, ${ }^{26}$ which do not determine the periodontal status but represent a greater risk of getting the disease, were considerably higher in patients with AVE. In connection with mean platelet volume, a significant difference was observed between patients with acute vascular events (cases) and those without it (controls), both groups with periodontitis, in favor of the latter. When controls with periodontitis were analyzed as compared to those without, MPV was significantly higher in the former. Lastly, two groups of patients with and without periodontal disease were evaluated, whether or not they had acute vascular events; patients with POD were found to have a higher MPV than subjects without periodontitis. In contrast to the study by Androsz-Kowalska $\mathrm{O}$ et al. ${ }^{27}$ where they found a difference in MPV between patients with coronary heart disease to those without it, ${ }^{27}$ our findings not only did not shown those differences but also may point to the fact that, even though platelet volume might indicate a platelet activation state over a systemic inflammatory condition, it is probably more related to the active periodontal disease as an inflammatory disease than to the vascular disease expressed through events such as ACS, IschS or PAD. This may be an indicator of either a subclinical vascular disease in patients with POD and a rise in the mean platelet volume, or the presence of low plasma vitamin D levels, as it is demonstrated, which are connected with a higher MPV. ${ }^{28}$ The importance of identifying this association lies in the existing evidence that high MPV may be a predictor of severity or mortality not only in the ischemic heart disease, ${ }^{29}$ ischemic cerebral disease ${ }^{30}$ but also in patients with congestive heart failure as recently was published by Czerniuk MR et al. ${ }^{31}$

\section{Conclusion}

The study showed a strong association between periodontal disease and vascular events in their acute phase and achieves interesting results to continue along similar lines of research with appropriate study designs for the aims proposed. However, the study could not demonstrate that patients with acute vascular events have high mean platelet volume in contrast to those without AVE. On the other hand, the study demonstrates the association between the presence of periodontitis and high mean platelet volume whether or not the patients had acute vascular events. Mean platelet volume might be an indicator of a related disease perhaps periodontitis, which is likely to trigger or perpetuate the vascular disease.

\section{Funding}

None. 


\section{Acknowledgments}

To biochemists Mónica Alonso and Sol Mitre for their invaluable contribution to sample collection and testing. To Dr. Alejandro Schroeder for approving part of the grant for this study and the use of the facilities at Fundación Médica de Río Negro y Neuquén (Medical Foundation of Río Negro and Neuquén) and Clínica Radiológica del Sur SA (South Radiological Clinic). To Pablo Zabala and Ezequiel Soloaga from Fundación Médica de Río Negro y Neuquén. To Pablo Granados, Julia Cabanne and Jorge Terenzi, medical students at the National University of Comahue. Also, to Ivana Dellapittima, Katia Gonzalez and Mayra Melinguer, dentistry students at the National University of Río Negro, for their collaboration on the fieldwork.

\section{Conflicts of interest}

The authors claim not to have any conflict of interest.

\section{References}

1. James B, Sara L, Khosrow Heidari, et al. Indicators for chronic disease surveillance. Centers for Disease Control and Prevention, MMWR. 2015;53(No. RR-11):1-89.

2. Bhatt DL, Steg PG, Ohman EM, et al. International Prevalence, Recognition, and Treatment of Cardiovascular Risk Factors in Outpatients with Atherothrombosis. JAMA. 2006;295(2):180-189.

3. Falk E. Pathogenesis of atherosclerosis. J Am Coll Cardiol. 2006;47(8 Suppl):C7-C12.

4. Virmani R, Kolodgie FD, Burke AP, et al. Lessons from sudden coronary death: a comprehensive morphological classification scheme for atherosclerotic lesions. Arterioscler Thromb Vasc Biol. 2000;20(5):1262-1275.

5. Buffon A, Biasucci LM, Liuzzo G, et al. Widespread coronary inflammatioin in unstable angina. $N$ Engl J Med. 2002;347(1):5-12.

6. Rioufol G, Finet G, Ginon I, et al. Multiple atherosclerotic plaque rupture in acute coronary syndrome: a three-vessel intravascular ultrasound study. Circulation. 2002;106:804-808.

7. Kannel WB, Dawber TR, Kagan A, et al. Factors of risk in the development of coronary heart disease - six-year follow-up experience: the Framingham Study. Ann Intern Med. 1961;55:33-50.

8. Spagnoli LG, Bonanno E, Mauriello A, et al. Multicentric inflammation in epicardial coronary arteries of patients dying of acute myocardial infarction. J Am Coll Cardiol. 2002;40(9):1579-1588.

9. Bahekar AA, Singh S, Saha S, et al. The prevalence and incidence of coronary heart disease is significantly increased in periodontitis: a metaanalysis. Am Heart J. 2007;154(5):830-837.

10. Alonso-González R, Pérez-Hernández A, Silvestre-Rangil J, et al. Cardiovascular disease versus periodontal disease: Chronic systemic infection as a link. J Clin Exp Dent. 2011;3(5):e476-e482.

11. Nakajima T, Honda T, Domon H, et al. Periodontitis-associated upregulation of systemic inflammatory mediator level may increase the risk of coronary heart disease. J Periodontal Res. 2010;45:116-22.

12. Lockhart PB, Bolger AF, Papapanou PN, et al. Periodontal disease and atherosclerotic vascular disease: does the evidence support an independent association? a scientific statement from the American Heart Association. Circulation. 2012;125(10):2520-2544.

13. Endler G, Klimesch A, Sunder-Plassmann H, et al. Mean platelet volume is an independent risk factor for myocardial infarction but not for coronary artery disease. Br J Haematol. 2002;117(2):399-404.
14. Santimone I, Di Castelnuovo AF, De Curtis A, et al. White Blood Cells Count, Sex and Age Are Major Determinants of Platelet Indices Heterogeneity in An Adult General Population: Results From The MOLISANI Project. Haematologica. 2011;96(8):1180-1188.

15. Tavil Y, Sen N, Yazici H, et al. Coronary heart disease is associated with mean platelet volume in type 2 diabetic patients. Platelets. 2010;21(5):368-372.

16. O’Malley T, Langhorne P, Elton RA, et al. Platelet size in stroke patients. Stroke. 1995;26(6):995-999.

17. Wang X, Meng H, Xu L, et al. Mean platelet volume as an inflammatory marker in patients with severe periodontitis. Platelets. 2015;26(1):67-71.

18. Ramesh A, Thomas B, Rao A. Evaluation of the association between chronic periodontitis and acute coronary syndrome: A case control study. J Indian Soc Periodontol. 2013;17(2):210-213.

19. Grundy SM, Pasternak R, Greenland P, et al. Assessment of Cardiovascular Risk by Use of Multiple-Risk-Factor Assessment Equations. Circulation. 1999;100(13):1481-1492.

20. Amsterdam EA, Wenger NK, Brindis RG, et al. 2014 AHA/ACC guideline for the management of patients with non-ST-elevation acute coronary syndromes. Circulation. 2014;130(25):2354-2394.

21. Brown W, Trost S, Bauman A, et al. Test-retest reliability of four physical activity measures used in population surveys. J Sci Med Sport. 2004;7(2):205-215.

22. Silness J, Löe H. Periodontal Disease in Pregnancy II. Correlation Between Oral Hygiene and Periodontal Condition. Acta Odontol Scand. 1964;22:121-135.

23. Nakajima T, Honda $T$, Domon $H$, et al. Periodontitis-associated upregulation of systemic inflammatory mediator level may increase the risk of coronary heart disease. J Periodontal Res. 2010;45(1):116-122.

24. Lockhart PB, Bolger AF, Papapanou PN, et al. Periodontal disease and atherosclerotic vascular disease: does the evidence support an independent association? a scientific statement from the American Heart Association. Circulation. 2012;125(20):2520-2544.

25. Endler G, Klimesch A, Sunder-Plassmann H, et al. Mean platelet volume is an independent risk factor for myocardial infarction but not for coronary artery disease. Br J Haematol. 2002;117(2):399-404.

26. Brown LJ, Brunelle JA, Kingman A. Periodontal status in the United States, 1988-1991: Prevalence, extent, and demographic variation. J Dent Res. 1996;75:672-783.

27. Androsz-Kowalska O, Jankowski K, Rymarczyk Z, et al. Correlation between clinical parameters of periodontal disease and mean platelet volume in patients with coronary artery disease: a pilot study. Kardiol Pol. 2013;71(6):600-605.

28. Cumhur Cure M, Cure E, Yuce S, et al. Mean platelet volume and vitamin D level. Ann Lab Med. 2014;34(2):98-103.

29. Slavka G, Perkmann T, Haslacher H, et al. Mean Platelet Volume May Represent a Predictive Parameter for Overall Vascular Mortality and Ischemic Heart Disease. Arterioscler Thromb Vasc Biol. 2011;31(5):1215-1218

30. Bath P, Algert C, Chapman N, et al. Association of Mean Platelet Volume with Risk of Stroke Among 3134 Individuals with History of Cerebrovascular Disease. Stroke. 2004;35:622-626.

31. Czerniuk MR, Bartoszewicz Z, Dudzik-Niewiadomska I, et al. Simple platelets markers: Mean platelet volume and congestive heart failure coexistent with periodontal disease. Pilot Studies. 2017. 